\title{
Mutant IDH and non-mutant chondrosarcomas display distinct cellular metabolomes
}

Sinthu Pathmanapan 1,2, Olga Ilkayeva ${ }^{3,4}$, John T. Martin ${ }^{5}$, Adrian Kwan Ho Loe', Hongyuan Zhang ${ }^{5}$, Guo-Fang Zhang ${ }^{4}$, Christopher B. Newgard ${ }^{3,4}$, Jay S. Wunder ${ }^{6}$ and Benjamin A. Alman ${ }^{5^{*}}$ (I)

\begin{abstract}
Background: Majority of chondrosarcomas are associated with a number of genetic alterations, including somatic mutations in isocitrate dehydrogenase 1 (IDH1) and IDH2 genes, but the downstream effects of these mutated enzymes on cellular metabolism and tumor energetics are unknown. As IDH mutations are likely to be involved in malignant transformation of chondrosarcomas, we aimed to exploit metabolomic changes in IDH mutant and nonmutant chondrosarcomas.
\end{abstract}

Methods: Here, we profiled over 69 metabolites in 17 patient-derived xenografts by targeted mass spectrometry to determine if metabolomic differences exist in mutant IDH1, mutant IDH2, and non-mutant chondrosarcomas. UMAP (Uniform Manifold Approximation and Projection) analysis was performed on our dataset to examine potential similarities that may exist between each chondrosarcoma based on genotype.

Results: UMAP revealed that mutant IDH chondrosarcomas possess a distinct metabolic profile compared with non-mutant chondrosarcomas. More specifically, our targeted metabolomics study revealed large-scale differences in organic acid intermediates of the tricarboxylic acid (TCA) cycle, amino acids, and specific acylcarnitines in chondrosarcomas. Lactate and late TCA cycle intermediates were elevated in mutant IDH chondrosarcomas, suggestive of increased glycolytic metabolism and possible anaplerotic influx to the TCA cycle. A broad elevation of amino acids was found in mutant IDH chondrosarcomas. A few acylcarnitines of varying carbon chain lengths were also elevated in mutant IDH chondrosarcomas, but with minimal clustering in accordance with tumor genotype. Analysis of previously published gene expression profiling revealed increased expression of several metabolism genes in mutant IDH chondrosarcomas, which also correlated to patient survival.

Conclusions: Overall, our findings suggest that IDH mutations induce global metabolic changes in chondrosarcomas and shed light on deranged metabolic pathways.

Keywords: Chondrosarcoma, Metabolism, Mutant IDH, Cancer, Genetic mutation, Amino acids, Acylcarnitines, TCA cycle, Glycolysis

\footnotetext{
* Correspondence: ben.alman@duke.edu

${ }^{5}$ Department of Orthopaedic Surgery, Duke University, 311 Trent, Durham, NC 27710, USA

Full list of author information is available at the end of the article
}

C C The Author(s). 2021 Open Access This article is licensed under a Creative Commons Attribution 4.0 International License, which permits use, sharing, adaptation, distribution and reproduction in any medium or format, as long as you give appropriate credit to the original author(s) and the source, provide a link to the Creative Commons licence, and indicate if changes were made. The images or other third party material in this article are included in the article's Creative Commons licence, unless indicated otherwise in a credit line to the material. If material is not included in the article's Creative Commons licence and your intended use is not permitted by statutory regulation or exceeds the permitted use, you will need to obtain permission directly from the copyright holder. To view a copy of this licence, visit http://creativecommons.org/licenses/by/4.0/ The Creative Commons Public Domain Dedication waiver (http://creativecommons.org/publicdomain/zero/1.0/) applies to the data made available in this article, unless otherwise stated in a credit line to the data. 


\section{Background}

Over the past decade, reprogramming of cellular metabolism has gained attention as an emerging hallmark of cancer [1], but metabolic studies of bone and cartilage tumors have been limited. Chondrosarcomas are the most common malignancy of cartilage. They are associated with a number of genetic alterations including somatic mutations in isocitrate dehydrogenase 1 (IDH1) and IDH2 [2, 3]. IDH mutations have been identified in 38$70 \%$ of chondrosarcoma cases [4] and for this reason, $I D H$ has been identified as a potential driver mutation. However, $52-87 \%$ of the benign precursor lesion, enchondromas, also harbor IDH mutations [4], suggesting that the abnormal $I D H$ genes could potentially orchestrate early events in chondrosarcoma formation. These mutations are also found in several neoplasms including gliomas, glioblastomas, and acute myeloid leukemia [3].

$I D H 1$ and IDH2 are located in the cytosol and mitochondria respectively, and catalyze the oxidative decarboxylation of isocitrate to $\alpha$-ketoglutarate $(\alpha-K G)[5]$. The mutant enzyme loses its ability to convert isocitrate to $\alpha-K G$, and instead gains a neomorphic function to produce D-2-hydroxyglutarate (D-2HG), which has been referred to as an "oncometabolite" [6]. IDH mutations have been reported to be involved in epigenetic remodeling [7], stabilization of hypoxia-inducible factor 1a $(\mathrm{HIF} 1 \alpha)[8]$, and regulation of alternative energetic pathways such as fatty acid metabolism [9].

Here, we have utilized a targeted metabolomics approach [10] to uncover potential metabolic differences between mutant $I D H$ and non-mutant chondrosarcomas. Specifically, we compared metabolomic profiles of $4 \mathrm{IDH}$ non-mutant chondrosarcomas with 8 mutant $I D H 1$ and 5 mutant IDH2 chondrosarcomas (Table 1) using targeted gas chromatography-mass spectrometry (GC/MS) and flow-injection tandem mass spectrometry (MS/MS) methods [10]. By measuring a large number of metabolites, profiles of chondrosarcoma tissues were constructed to comprehensively cover a number of biological processes and metabolic pathways. This approach has been used by others to identify novel metabolic biomarkers and mechanisms in cancer, stem cells, and healthy tissues [10-13]. Our results highlight a number of metabolomic alterations and differentially regulated metabolic pathways in mutant $I D H$ compared with non-mutant chondrosarcomas.

\section{Methods}

The aim of this study was to assess the metabolic differences between mutant $I D H$ and non-mutant chondrosarcomas. To identify and quantify metabolites in patient-derived chondrosarcoma xenograft tissue extracts, we performed targeted GC/MS or flow-injection MS/MS methods.

\section{Patient-derived xenograft establishment}

All human chondrosarcoma samples were handled according to the ethical guidelines of the host institutions. With institutional review board (IRB) approval, human chondrosarcoma tumor samples were obtained fresh from surgery. Within $24 \mathrm{~h}$, tumors were cut into $2-\mathrm{mm}^{3}$

Table 1 Sequence results and clinicopathological information of chondrosarcomas used for metabolomics analysis

\begin{tabular}{|c|c|c|c|c|c|}
\hline Genotype & Mutation & Tumor grade & Chondrosarcoma subtype & Metastatic status & Recurrence \\
\hline $\mathrm{IDH1/2-WT}$ & WT & 2 & Central & No Metastasis & No \\
\hline IDH1/2-WT & WT & 2 & Central & No Metastasis & No \\
\hline IDH1/2-WT & WT & 2 & Central & No Metastasis & No \\
\hline IDH1/2-WT & WT & 1 & Central & No Metastasis & No \\
\hline Mutant IDHI & $\mathrm{R} 132 \mathrm{C}$ & 3 & Central & Later Metastasis & Yes \\
\hline Mutant IDHI & $\mathrm{R} 132 \mathrm{C}$ & 3 & Central & Later Metastasis & Yes \\
\hline Mutant IDH1 & $\mathrm{R} 132 \mathrm{C}$ & 2 & Central & No Metastasis & No \\
\hline Mutant IDH1 & $\mathrm{R} 132 \mathrm{C}$ & 3 & Central & No Metastasis & No \\
\hline Mutant IDH1 & $\mathrm{R} 132 \mathrm{G}$ & 1 & Clear Cell & Later Metastasis & Yes \\
\hline Mutant IDH1 & R132G & 2 & Dedifferentiated & Later Metastasis & Yes \\
\hline Mutant IDHI & $\mathrm{R} 132 \mathrm{H}$ & 3 & Clear Cell & Metastasis at Presentation & No \\
\hline Mutant IDH1 & $\mathrm{R} 132 \mathrm{H}$ & 3 & Dedifferentiated & Metastasis at Presentation & No \\
\hline Mutant IDH2 & $\mathrm{R} 172 \mathrm{G}$ & 3 & Dedifferentiated & Later Metastasis & Yes \\
\hline Mutant IDH2 & $\mathrm{R} 172 \mathrm{M}$ & 3 & Dedifferentiated & Metastasis at Presentation & No \\
\hline Mutant IDH2 & $\mathrm{R} 172 \mathrm{M}$ & 3 & Dedifferentiated & Metastasis at Presentation & No \\
\hline Mutant IDH2 & $\mathrm{R} 172 \mathrm{~S}$ & 3 & Dedifferentiated & Later Metastasis & Yes \\
\hline Mutant $1 \mathrm{DH} 2$ & R172S & 3 & Dedifferentiated & Metastasis at Presentation & No \\
\hline
\end{tabular}


pieces in sterile conditions and were subcutaneously implanted into the flank region of interleukin-2 receptor gamma chain (gamma)-null NOD/SCID (NSG) mice. Tumors were excised once maximal tumor capacity of $2.5 \mathrm{~cm}$ was reached. An important consideration in studying tumor metabolomics in patients is variability due to differences in diet, time of surgery, and the presence of medical comorbidities. To control for these patient-specific factors, we established patient-derived chondrosarcoma xenografts in immunodeficient mice. This allowed us to study chondrosarcoma tumor metabolism by eliminating these confounding factors.

\section{Sequencing}

Chondrosarcoma xenograft tumors were genotyped by extracting genomic DNA using the DNeasy Blood and Tissue Kit (Qiagen) according to the manufacturer's protocol. Sanger sequencing was performed to analyze the samples for $I D H 1$ and $I D H 2$ mutations after PCR amplification on exon 4 of $I D H 1$ and $I D H 2$ (Table 1). Sequencing results were analyzed on 4Peaks software to confirm mutational status of each tumor.

\section{Metabolite profiling}

To prepare tumors for metabolomics analysis, tumors were pulverized using a prechilled cell crusher in liquid nitrogen. Approximately $50 \mathrm{mg}$ of tumor tissue was added into a prechilled vial and homogenizing solvent (50\% aqueous acetonitrile containing $0.3 \%$ formic acid) was added to achieve the desired tissue concentration of $50 \mathrm{mg} / \mathrm{ml}$. Mini-BeadBeater Zirconia-Silicate Beads (BioSpec Products), $0.5 \mathrm{~mm}$, were added to the vial to aid with homogenization. Samples were homogenized using Mini-BeadBeater (BioSpec Products) set to a frequency of 30 oscillations/s for $2 \mathrm{~min}$. Metabolic quantification was performed by gas chromatography-mass spectrometry (GC/MS) or flow-injection tandem mass spectrometry (MS/MS) as described previously $[14,15]$. Amino acids, acylcarnitines, and organic acids were analyzed using the stable isotope dilution technique. Amino acids and acylcarnitine measurements were made by flow-injection tandem mass spectrometry using sample preparation methods described previously [16, 17]. The data were acquired using a Waters TQD mass spectrometer equipped with an Acquity ${ }^{\mathrm{TM}}$ UPLC system and controlled by MassLynx 4.1 operating system (Waters, Milford, MA). Organic acids were quantified using methods described previously [18] employing Trace Ultra GC coupled to ISQ MS operating under Xcalibur 2.2 (Thermo Fisher Scientific, Austin, TX). Data analysis of metabolites was performed using Student's $t$-test $(p<$ 0.05).

\section{UMAP analysis}

To evaluate relative similarities between each chondrosarcoma, data was processed using Uniform Manifold Approximation and Projection [19] (UMAP) for dimensional reduction and visualization. In total, 7 organic acids, 17 amino acids, and 45 acylcarnitines were screened and separate UMAP projections were performed for each metabolite group (i.e., three groups with 17 tumors and 7, 17, or 45 dimensions). First, data was pre-processed by scaling the mean and variance $(\mu=0$ and $\sigma=1)$. Then, UMAP was performed for $2 \mathrm{D}$ visualization ( $\mathrm{R}$ version 4.0.0 [20]); Package "Seurat" (Stuart, Butler, Hoffman, et al., 2019)-function RunUMAP; nearest neighbors: 15 , minimum distance: 0.7 , distance metric: Euclidean). After UMAP projection, tumors were labeled based on their genotype and evaluated qualitatively.

\section{Carbon isotope labeling}

Chondrosarcoma cells were cultured in DMEM with $1000 \mathrm{mg} / \mathrm{L}^{13} \mathrm{C}_{6}$-Glucose in 6 -cm cell culture plates for 6 h. A total of $500 \mu \mathrm{L}$ methanol was used to extract metabolites from each plate. After centrifuging at 12000 $\mathrm{rpm}$ for $15 \mathrm{~min}$, supernatant was dried at $37{ }^{\circ} \mathrm{C}$. The dried residues were resuspended in $25 \mu \mathrm{L}$ of methoxylamine hydrochloride ( $2 \%(\mathrm{w} / \mathrm{v})$ in pyridine) and incubated at $40{ }^{\circ} \mathrm{C}$ for $1.5 \mathrm{~h}$ in a heating block. After brief centrifugation, $35 \mu \mathrm{L}$ of MTBSTFA $+1 \%$ TBDMS was added, and the samples were incubated at $60{ }^{\circ} \mathrm{C}$ for $30 \mathrm{~min}$. The derivatized samples were centrifuged for $5 \mathrm{~min}$ at $20,000 \times g$, and the supernatants were transferred to GC vials for GC-MS analysis. A modified GC-MS method was employed [21]. The injection volume was $1 \mu \mathrm{L}$, and samples were injected in splitless mode. GC oven temperature was held at $80{ }^{\circ} \mathrm{C}$ for $2 \mathrm{~min}$, increased to $280{ }^{\circ} \mathrm{C}$ at $7{ }^{\circ} \mathrm{C} / \mathrm{min}$, and held at $280{ }^{\circ} \mathrm{C}$ for a total run time of $40 \mathrm{~min}$. GC-MS analysis was performed on an Agilent 7890B GC system equipped with a HP-5MS capillary column ( $30 \mathrm{~m}, 0.25 \mathrm{~mm}$ i.d., $0.25-\mu \mathrm{m}$ phase thickness; Agilent I\&W Scientific, Santa Clara, CA), connected to an Agilent 5977A Mass Spectrometer operating under ionization by electron impact (EI) at 70 $\mathrm{eV}$. Helium flow was maintained at $1 \mathrm{~mL} / \mathrm{min}$. The source temperature was maintained at $230{ }^{\circ} \mathrm{C}$, the MS quad temperature at $150{ }^{\circ} \mathrm{C}$, the interface temperature at $280{ }^{\circ} \mathrm{C}$, and the inlet temperature at $250{ }^{\circ} \mathrm{C}$. Mass spectra were recorded in mass scan mode with $\mathrm{m} / \mathrm{z}$ from 50 to 700 .

\section{${ }^{13} \mathrm{C}$-based stable isotope analysis}

$\mathrm{M} 0, \mathrm{M} 1, \ldots, \mathrm{M} n$ refers to the isotopologues containing $n$ heavy atoms in a molecule. The stable isotope distribution of individual metabolites was measured by GC-MS as described above. The isotopologue enrichment or 
labeling in this work refers to the corrected isotope distribution [22, 23]. To determine the flux from citrate/ isocitrate to $\alpha$-ketoglutarate, we normalized the $\%$ of ${ }^{13} \mathrm{C}$ - $\alpha$-ketoglutarate to the percentage of ${ }^{13} \mathrm{C}$-citrate/ isocitrate.

\section{Gene expression profiling data analysis}

Chondrosarcoma gene expression data was previously published by another group in their identification of genes that determine disease progression [24]. Their mRNA microarray data and chondrosarcoma sample information [25] E-MTAB-7264 (https://www.ebi.ac.uk/ arrayexpress/experiments/E-MTAB-7265/) were downloaded from the European Bioinformatics Institute database [26] [PMID: 31604924]. Microarray data was normalized using the RMA algorithm with the "oligo" package [PMID: 20688976]. Gene expression data was further scaled for the generation of heatmaps. For survival analysis, samples were grouped into high/low expression based on median gene expression and plotted using the "survminer" package.

\section{Results \\ Mutant IDH1 and IDH2 chondrosarcomas display an elevation of glycolytic and TCA cycle intermediates compared with non-mutant chondrosarcomas}

To examine whether mutant $I D H$ chondrosarcomas display metabolomic differences in comparison with nonmutant chondrosarcomas, we used gas chromatography/ mass spectrometry (GC/MS) to profile 7 organic acids generated by glycolysis and the TCA cycle. Mutant $I D H 1$ and $I D H 2$ chondrosarcomas displayed a 3.5-fold elevation of lactate (Fig. 1b), coupled with a trend for increase of pyruvate (Fig. 1a), suggestive of enhanced glycolytic metabolism of glucose in mutant $I D H$ chondrosarcomas.

Five TCA cycle intermediates were also quantified to determine if differences may exist in mitochondrial carbon cycling. Three intermediates generated in the latter part of the TCA cycle, succinate, fumarate, and malate were increased in mutant IDH1 and IDH 2 chondrosarcomas compared with non-mutant chondrosarcomas (Fig. 1e-g). A different and more complex profile was found for the early TCA cycle intermediates-citrate and $\alpha$-KG trended to increase in mutant $I D H 1$ but decrease in mutant IDH2 chondrosarcomas (Fig. 1c, d). These differences may reflect the role of IDH1 in the cytosol as a source of NADPH for de novo lipogenesis [27] and other biosynthetic pathways, whereas $I D H 2$ participates both in oxidative flux of $\alpha$-ketoglutarate to the later TCA cycle intermediates, as well as reductive carboxylation of $\alpha$-ketoglutarate to isocitrate and citrate in the mitochondria $[28,29]$.
Activation of reductive TCA cycle metabolism of glutamine and glutamate via conversion to $\alpha$-ketoglutarate by the glutamate dehydrogenase reaction and flux to citrate via $I D H 2$ has been invoked as a pathway for synthesis of biomass in growing cancer cells [29-31]. If $I D H 2$ mutation impairs reductive flux of glutamine to citrate in chondrocytes, this may help to explain why citrate and $\alpha$-ketoglutarate are depleted in mutant IDH2 chondrosarcomas (Fig. 1c, d; Supplemental Figure 2). Perhaps related, it has been suggested that citrate and $\alpha$ ketoglutarate levels are influenced by multiple events limiting entry of carbon into the TCA cycle [30, 32, 33] including reduced mitochondrial $\mathrm{NAD}^{+} / \mathrm{NADH}$ ratio [30], reduced glutamine metabolism [30, 33], and inactivation of pyruvate dehydrogenase in hypoxic tumors [32].

To examine how mutant $I D H$ alters flux through the TCA cycle, we traced $\left[{ }^{13} \mathrm{C}_{6}\right]$-labeled glucose. ${ }^{13} \mathrm{C} \alpha$-KG labeling was reduced at $6 \mathrm{~h}$ in mutant $I D H 1$ and $I D H 2$ chondrosarcoma cells based on measurements of citrate (M2) and pyruvate (M3) labeling (Supplemental Figure 2). This result clarifies that carbon flux through mutant $I D H$ is impaired, explaining depleted $\alpha-K G$ levels (Fig. $1 \mathrm{~d})$ in mutant $I D H 2$ chondrosarcomas.

We next performed UMAP clustering analysis [19] on our metabolite dataset to examine if relative similarities exist between each chondrosarcoma genotype group (Supplemental Figure 1). UMAP projections performed on 7 organic acids revealed distinct clustering patterns in non-mutant and mutant IDH chondrosarcomas (Supplemental Figure 1A). Although organic acids in mutant IDH1 and mutant IDH2 chondrosarcomas did not cluster separately from one another, they clustered separately from the non-mutant group (Supplemental Figure 1A). UMAP projections reveal that chondrosarcomas of identical genotypes exhibit similar organic acid profiles. Taken together, our data is consistent with the notion that mutant $I D H$ chondrosarcomas not only display high glycolytic and TCA cycle intermediates for energy production and maintenance but also display a distinct organic acid profile in comparison with non-mutant chondrosarcomas.

\section{Global elevation of amino acids in mutant IDH chondrosarcomas in comparison with non-mutant chondrosarcomas}

We next measured levels of 17 amino acids by MS/MS and constructed amino acid profiles of each patient tumor. We aimed to characterize overlapping and/or distinct patterns of expression in mutant $I D H$ and nonmutant chondrosarcomas. Irrespective of mutational status, glycine (Gly), glutamate (Glu), glutamine (Gln), and alanine (Ala) were the most abundant amino acids in chondrosarcomas (Fig. 2a-c). This observation is 


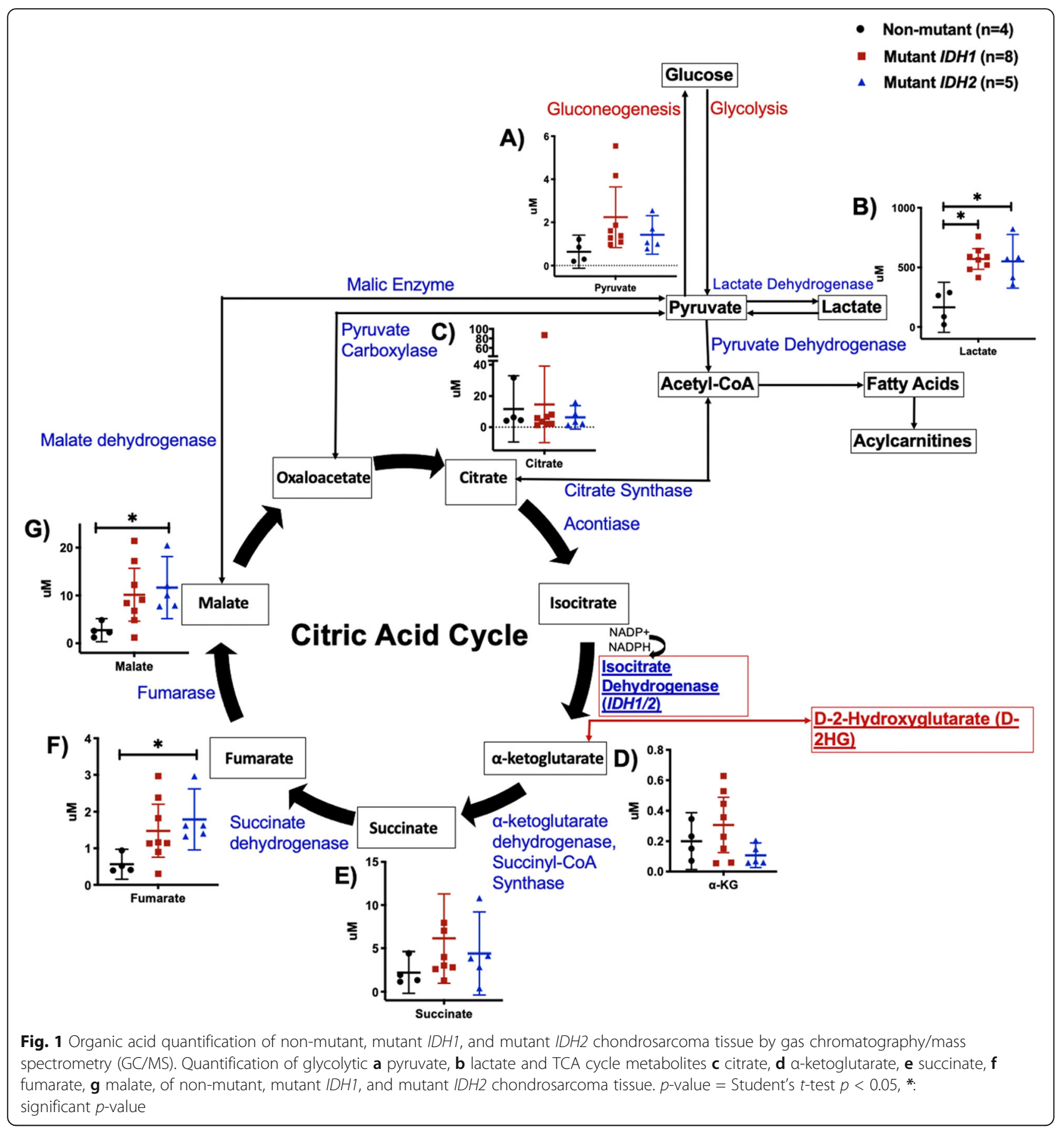

supported by the sharp peak concentrations as indicated from radar profiles of chondrosarcoma tissues (Fig. 2ac).

A broad elevation of amino acids was observed in mutant $I D H$ chondrosarcomas compared with non-mutant tumors (Table 2, Fig. 2d). Twelve of the 17 amino acids measured (Val, Met, His, Gly, Pro, Ser, Asn, Ala, Leu/Ile, Phe, Tyr, and Orn) were significantly elevated in mutant IDH1 chondrosarcomas. These same 12 amino acids exhibited clear trends to increase in mutant $I D H 2$ chondrosarcomas, although only 5 of these (Gly, Gln, Pro, Ser, and Asp) achieved statistical significance (Table 2, Fig. 2d). Mechanisms for this broad increase in amino acids are not revealed by our data, but two possibilities are that mutant $I D H$ may activate autophagy [34-36] or protein degradation [37, 38]. We used UMAP clustering analysis to determine if mutant IDH chondrosarcomas displayed distinct amino acid profiles compared with non-mutant chondrosarcomas (Supplemental Figure 1B). Although amino acid profiles of mutant IDH 


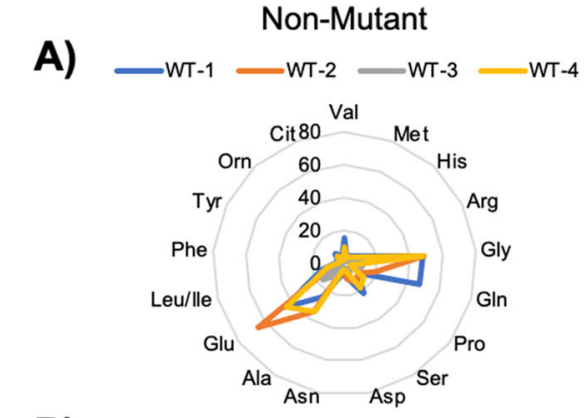

B)

Mutant IDH1
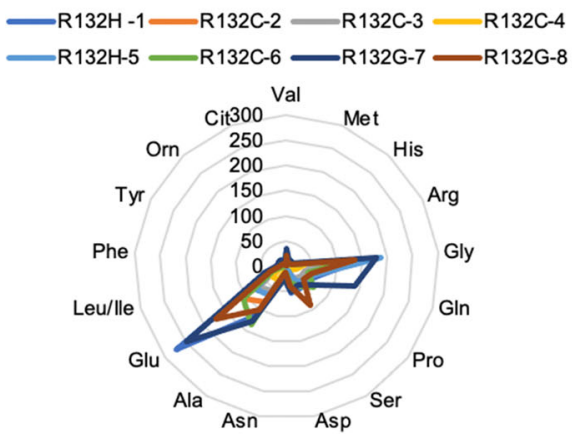

C)

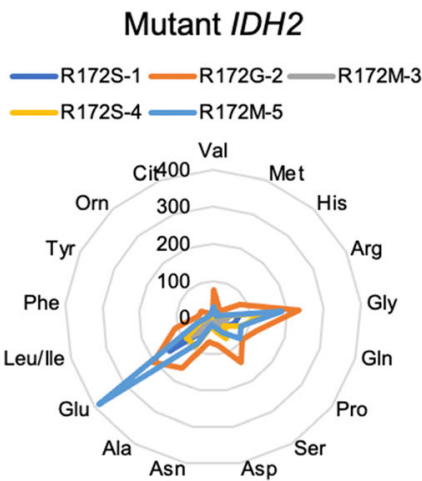

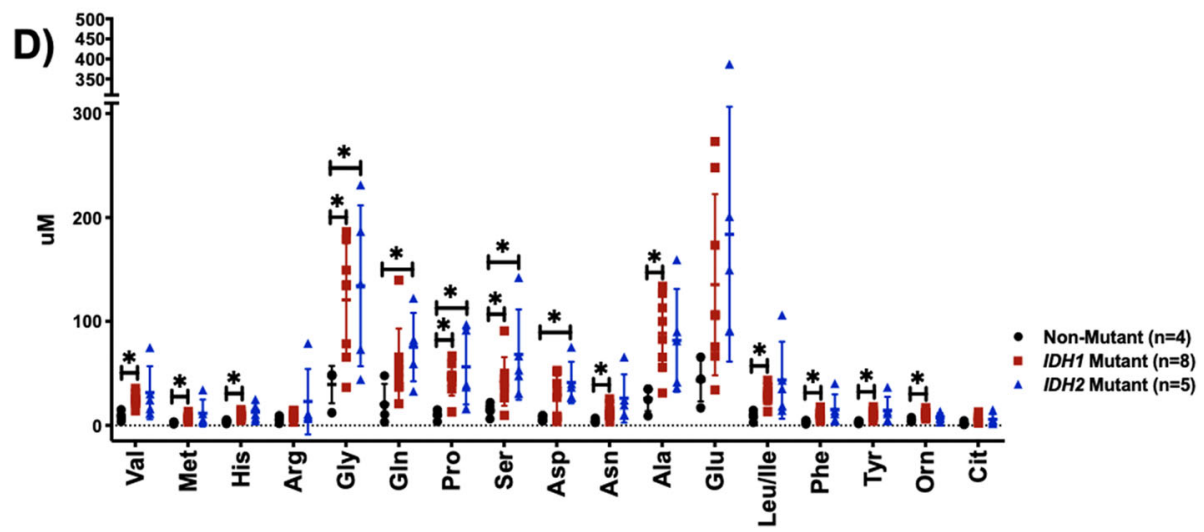

Fig. 2 Amino acid profiles of non-mutant, mutant IDH, and mutant IDH2 chondrosarcoma tissue quantified by mass spectrometry (MS/MS) display a global elevation in mutant IDH chondrosarcomas. Radar profiles of a non-mutant chondrosarcomas, $\mathbf{b}$ mutant IDH1 chondrosarcomas, $\mathbf{c}$ mutant IDH2 chondrosarcomas, $\mathbf{d}$ scatter plot of amino acids in each genotype of chondrosarcoma. $p$-value $=$ Student's $t$-test $p<0.05, *$ : significant $p$-value

chondrosarcomas clustered together, two mutant $I D H 1$ tumors clustered closely to the non-mutant cluster group (Supplemental Figure 1B).

\section{Amino acids involved in neoplastic energy-yielding pathways are elevated in mutant IDH chondrosarcomas} An abundant supply of amino acids is essential for tumors to sustain their proliferation through energy generation, nucleoside synthesis, protein synthesis, and cellular redox homeostasis [38]. For this reason, specific amino acids are coupled to neoplastic metabolic pathways [39]. Anaplerotic metabolism of amino acids regenerates key TCA cycle and glycolytic intermediates that enable carbon flow and ATP production in neoplastic cells. Further examination of our data suggests that several amino acids elevated by $I D H$ mutations are critical anaplerotic substrates.

Glutamine (Gln) and glutamate (Glu) are involved in glutamine metabolism to fuel reductive and oxidative TCA carbon cycling. Gln and Glu are converted to $\alpha$ ketoglutarate by the glutamate dehydrogenase reaction or by glutamate transamination. Gln levels trended to increase by 2.8 -fold in mutant IDH1 chondrosarcomas and achieved statistical significance in mutant $I D H 2$ chondrosarcomas (Table 2). Glu trended to increase by 3.2-fold and 4.3-fold in mutant IDH1 and mutant IDH2 
Table 2 Fold change analysis reveals global elevation of amino acids in mutant IDH chondrosarcomas in comparison with nonmutant chondrosarcomas

\begin{tabular}{|c|c|c|c|c|}
\hline $\begin{array}{l}\text { Amino } \\
\text { acid }\end{array}$ & $\begin{array}{l}\text { Fold change-mutant } I D H 1 \text { vs non-mutant } \\
\text { Chondrosarcoma }\end{array}$ & $p$-value & $\begin{array}{l}\text { Fold change-mutant } I D H 2 \text { vs non-mutant } \\
\text { Chondrosarcoma }\end{array}$ & $p$-value \\
\hline \multicolumn{5}{|c|}{ Glucogenic amino acids } \\
\hline Val & 2.8 & $0.0015^{*}$ & 3.6 & 0.1275 \\
\hline Met & 2.7 & $0.0156^{*}$ & 4.5 & 0.1989 \\
\hline His & 2.6 & $0.0071 *$ & 3.4 & 0.0703 \\
\hline Arg & 1.5 & 0.1729 & 3.8 & 0.3309 \\
\hline Gly & 3.1 & $0.0174^{*}$ & 3.4 & $0.0497^{*}$ \\
\hline Gln & 2.8 & 0.0950 & 3.7 & $0.0222 *$ \\
\hline Pro & 4.5 & $0.0021 *$ & 5.7 & $0.0404 *$ \\
\hline Ser & 2.8 & $0.0502 *$ & 4.5 & $0.0487^{*}$ \\
\hline Asp & 4.0 & 0.0639 & 5.8 & $0.0125 *$ \\
\hline Asn & 3.0 & $0.0340^{*}$ & 6.0 & 0.1083 \\
\hline Ala & 3.4 & $0.0080^{*}$ & 3.2 & 0.0659 \\
\hline Glu & 3.2 & 0.0680 & 4.3 & 0.0594 \\
\hline \multicolumn{5}{|c|}{ Ketogenic amino acids } \\
\hline Leu/ Ile & 3.2 & $0.0021 *$ & 4.8 & 0.1097 \\
\hline \multicolumn{5}{|c|}{ Glucogenic \& ketogenic amino acids } \\
\hline Phe & 3.3 & $0.0099^{*}$ & 4.9 & 0.1459 \\
\hline Tyr & 3.3 & $0.0068^{*}$ & 4.5 & 0.1403 \\
\hline \multicolumn{5}{|c|}{ Other amino acids } \\
\hline Orn & 2.0 & $0.0128^{*}$ & 1.5 & 0.2156 \\
\hline Cit & 2.8 & 0.0667 & 2.8 & 0.1950 \\
\hline
\end{tabular}

$p$-value $=t$-test $p<0.05$, italic, bold: significant in $t$-test

tumors respectively (Table 2). Additionally, proline (Pro), another critical amino acid metabolized via conversion to glutamate and was found to be elevated in mutant $I D H$ chondrosarcomas (Table 2). This suggests that mutant $I D H$ chondrosarcomas possess a greater reliance of glutamine metabolism.

Alanine (Ala), glycine (Gly), and serine (Ser), which are broadly elevated in mutant $I D H$ chondrosarcomas (Fig. 2d, Table 2), enter pathways of energy production via pyruvate, which in turn can be oxidized via pyruvate dehydrogenase or used as an anaplerotic substrate to generate oxaloacetate via pyruvate carboxylase. Aspartate (Asp) and asparagine (Asn), which are also elevated in mutant $I D H$ tumors (Fig. 2d, Table 2), act as anaplerotic substrates via their conversion to oxaloacetate by aspartate transaminase. A previous study identified that elevated Asp levels serve as an endogenous metabolite, supporting tumor growth via conversion of Asn to Asp by asparaginase activity [40] and maintenance of Asp pools by electron acceptor activity was found to support cell proliferation [41]. Interestingly, our profile suggests that Asn and Asp are crucial substrates for TCA cycle fueling in mutant $I D H$ chondrosarcomas.
Finally, the branched-chain amino acids (BCAAs), valine (Val), leucine (Leu), and isoleucine (Ile), have been implicated in metabolic reprogramming of neoplastic cells to promote cancer growth [42]. Individual Val and Leu/Ile levels were significantly elevated in mutant $I D H 1$ chondrosarcomas and trended to increase in mutant IDH2 chondrosarcomas (Table 2). The increase in Val and Leu/lle in mutant $I D H$ chondrosarcomas is associated with similar increases in their transamination byproducts Glu and Gln (Fig. 2a-d, Table 2).

\section{Acylcarnitine species are elevated in mutant IDH chondrosarcomas}

Acylcarnitines are generated by metabolism of fatty acids, glucose, and amino acids, and serve as cognate metabolites to acyl-CoAs generated in the mitochondria and cytosol [14]. We measured 45 independent acylcarnitine species ranging from 2 to 22 carbons in length, and with varying degrees of saturation by MS/MS. Even chain species ranging in length from $\mathrm{C} 6$ to $\mathrm{C} 22$ arise from fatty acid oxidation [14]. Odd chain species such as C3 (propionylcarnitine) and C5 (isovalerylcarnitine) are produced by amino acid catabolism, whereas C4 (butyrylcarnitine) can be derived from fatty acids or amino 
acids [14]. Acetylcarnitine (C2) is derived from acetyl$\mathrm{CoA}$, a common degradation product for many metabolic substrates. As in other tissues, acetylcarnitine (C2) is the most abundant acylcarnitine species in chondrosarcomas.

Interestingly, a number of long-chain lipid-derived acylcarnitines were found to be elevated in mutant $I D H$ chondrosarcomas compared with non-mutant tumors, including oleate (C18:1), palmitate (C16:0), linoleate (C18:2), and stearate (C18:0) (Fig. 3c, Table 3). Consistent with a profile of increased fatty acid oxidation was the increase in $\mathrm{C} 4-\mathrm{OH}$ acylcarnitine (beta-hydroxy butyryl AC) in mutant $I D H$ chondrosarcomas (Fig. 3a, Table 3). C3, C4, and C5 acylcarnitines derived from amino acid catabolism also trended higher in mutant IDH chondrosarcomas (Fig. 3a, Table 3).

To examine relative differences in acylcarnitine metabolism between mutant IDH and non-mutant chondrosarcomas, we compared means of acylcarnitine species in mutant $I D H$ tumors to non-mutant tumors and calculated concentration fold changes (Table 3). From this analysis, we found medium- and long-chain lipid-derived acylcarnitines, C10, C16, and C16:1-OH/C14:1-DC significantly elevated in mutant IDH1 chondrosarcomas

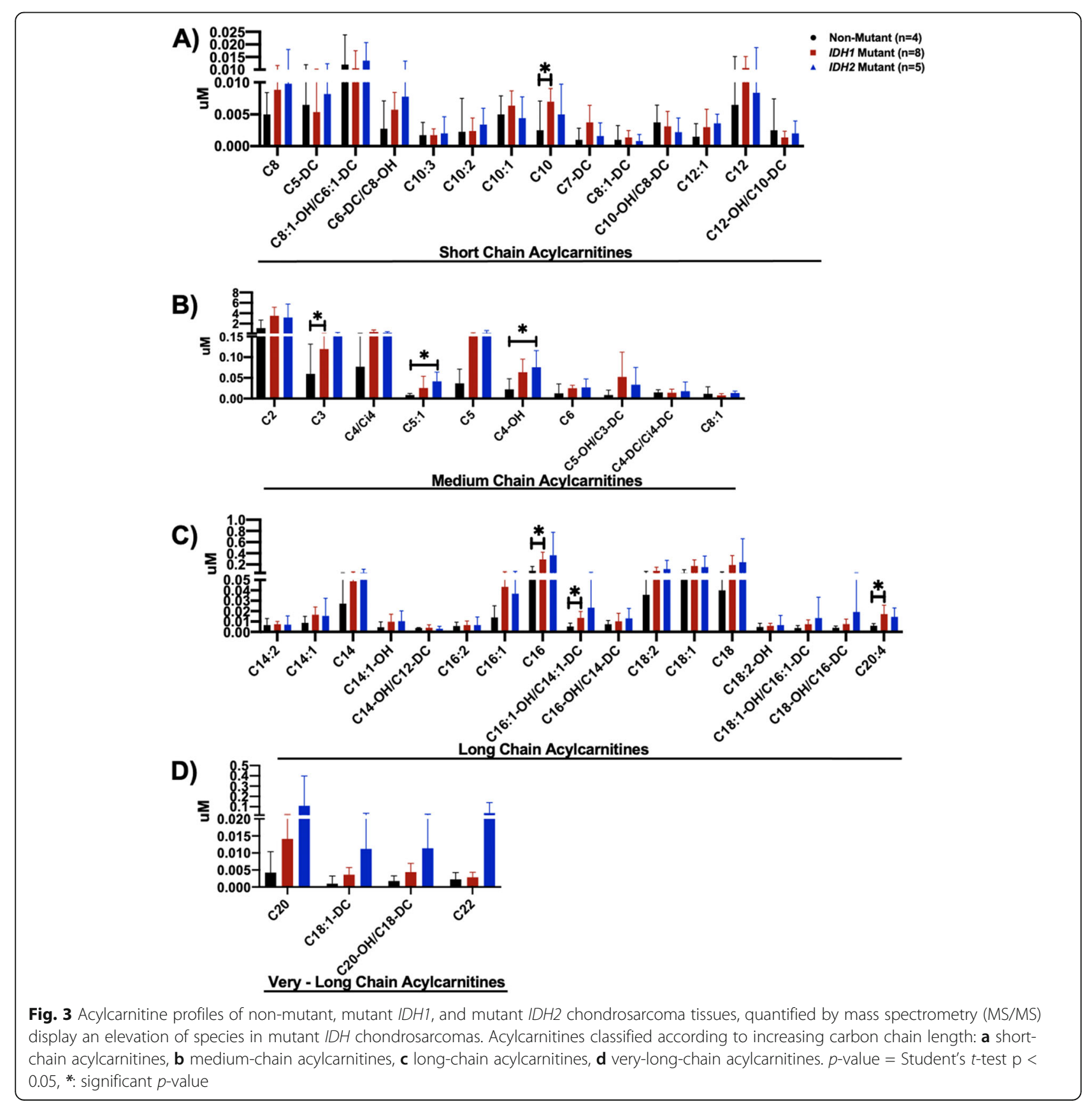


Table 3 Fold change analysis reveals elevated levels of acylcarnitine species in mutant IDH chondrosarcomas

\begin{tabular}{llr}
$\begin{array}{l}\text { Lipid profiling } \\
\text { acylcarnitines }\end{array}$ & $\begin{array}{l}\text { Acylcarnitine } \\
\text { length }\end{array}$ & $\begin{array}{l}\text { Fold } \\
\text { mut }\end{array}$ \\
\hline Acetyl-CoA derivative & & \\
C2 & SC-AC & 3.0 \\
Amino acid derivatives-Odd chain species \\
C3 & SC-AC & 2.0 \\
C5:1 & SC-AC & 3.0 \\
C5 & SC-AC & 4.3 \\
C5-OH/C3-DC & SC-AC & 6.2 \\
C5-DC & MC-AC & 0.9 \\
C7-DC & MC-AC & 3.7
\end{tabular}

Fatty acid derivatives_even chain species

\begin{tabular}{|c|c|}
\hline $\mathrm{C} 6$ & SC-AC \\
\hline C $8: 1$ & SC-AC \\
\hline $\mathrm{C} 8$ & MC-AC \\
\hline C8:1-OH/C6:1-DC & MC-AC \\
\hline $\mathrm{C} 6-\mathrm{DC} / \mathrm{C} 8-\mathrm{OH}$ & MC-AC \\
\hline $\mathrm{C} 10: 3$ & MC-AC \\
\hline $\mathrm{C} 10: 2$ & MC-AC \\
\hline C10:1 & MC-AC \\
\hline $\mathrm{C} 10$ & MC-AC \\
\hline C8:1-DC & MC-AC \\
\hline $\mathrm{C} 10-\mathrm{OH} / \mathrm{C} 8-\mathrm{DC}$ & $M C-A C$ \\
\hline C12:1 & MC-AC \\
\hline $\mathrm{C} 12$ & MC-AC \\
\hline $\mathrm{C} 12-\mathrm{OH} / \mathrm{C} 10-\mathrm{DC}$ & MC-AC \\
\hline C14:2 & LC-AC \\
\hline C14:1 & LC-AC \\
\hline C14 & LC-AC \\
\hline $\mathrm{C} 14: 1-\mathrm{OH}$ & LC-AC \\
\hline $\mathrm{C} 14-\mathrm{OH} / \mathrm{C} 12-\mathrm{DC}$ & LC-AC \\
\hline C16:2 & LC-AC \\
\hline C16:1 & LC-AC \\
\hline $\mathrm{C} 16$ & LC-AC \\
\hline $\begin{array}{l}\mathrm{C} 16: 1-\mathrm{OH} / \mathrm{C} 14: 1- \\
\text { DC }\end{array}$ & LC-AC \\
\hline $\mathrm{C} 16-\mathrm{OH} / \mathrm{C} 14-\mathrm{DC}$ & LC-AC \\
\hline C18:2 & LC-AC \\
\hline C18:1 & LC-AC \\
\hline $\mathrm{C} 18$ & LC-AC \\
\hline $\mathrm{C} 18: 2-\mathrm{OH}$ & LC-AC \\
\hline $\begin{array}{l}\text { C18:1-OH/C16:1- } \\
\text { DC }\end{array}$ & LC-AC \\
\hline $\mathrm{C} 18-\mathrm{OH} / \mathrm{C} 16-\mathrm{DC}$ & LC-AC \\
\hline C20:4 & LC-AC \\
\hline $\mathrm{C} 20$ & VLC-AC \\
\hline
\end{tabular}

$0.0510 \quad 2.8$

0.1189

old change-mutant IDH1 vs non-

mutant Chondrosarcoma

.0

3

0.9

3.7


Table 3 Fold change analysis reveals elevated levels of acylcarnitine species in mutant IDH chondrosarcomas (Continued)

\begin{tabular}{|c|c|c|c|c|c|}
\hline $\begin{array}{l}\text { Lipid profiling } \\
\text { acylcarnitines }\end{array}$ & $\begin{array}{l}\text { Acylcarnitine } \\
\text { length }\end{array}$ & $\begin{array}{l}\text { Fold change-mutant IDH1 vs non- } \\
\text { mutant Chondrosarcoma }\end{array}$ & $p$-value & $\begin{array}{l}\text { Fold change-mutant } I D H 2 \text { vs non- } \\
\text { mutant Chondrosarcoma }\end{array}$ & $p$-value \\
\hline C18:1-DC & VLC-AC & 3.3 & 0.0861 & 9.9 & 0.3591 \\
\hline $\mathrm{C} 20-\mathrm{OH} / \mathrm{C} 18-\mathrm{DC}$ & VLC-AC & 2.8 & 0.1026 & 7.0 & 0.2104 \\
\hline $\mathrm{C} 22$ & VLC-AC & 1.3 & 0.5074 & 15.7 & 0.4142 \\
\hline \multicolumn{6}{|c|}{ Amino acid \& fatty acid derivatives } \\
\hline $\mathrm{C} 4 / \mathrm{Ci} 4$ & SC-AC & 5.9 & 0.1180 & 3.7 & 0.0602 \\
\hline $\mathrm{C} 4-\mathrm{OH}$ & SC-AC & 2.9 & 0.0676 & 3.4 & $0.0193^{*}$ \\
\hline C4-DC/Ci4-DC & SC-AC & 1.0 & 0.9681 & 1.2 & 0.7348 \\
\hline
\end{tabular}

$p$-value $=t$-test $p<0.05$, italic, bold: significant in $t$-test

$S C-A C$ short-chain acylcarnitine

$M C-A C$ medium-chain acylcarnitine

LC-AC long-chain acylcarnitine

$V L C-A C$ very-long-chain acylcarnitine

compared with non-mutant chondrosarcomas (Table 3). The amino acid-derived acylcarnitines, $\mathrm{C} 5: 1$ and $\mathrm{C} 4-\mathrm{OH}$ were significantly elevated in mutant $I D H 2$ chondrosarcomas compared with non-mutant tumors (Table 3).

UMAP clustering analysis of 45 acylcarnitines revealed weak clustering of mutant $I D H$ and non-mutant chondrosarcomas (Supplemental Figure 1C). Although majority of mutant $I D H$ chondrosarcomas clustered away from the non-mutant group, these clusters were spatially weak clusters (Supplemental Figure 1C). Although there may be some similarities in acylcarnitine profile in mutant $I D H$ chondrosarcomas, these similarities are not as pronounced as clustering similarities noted in organic acid and amino acid profiles (Supplemental Figure 1AC). Clustering analysis of the complete dataset of 69 metabolites revealed distinct clustering of mutant $I D H$ chondrosarcomas from non-mutant tumors (Supplemental Figure 1D), emphasizing the notion that mutant IDH chondrosarcomas display a distinct metabolome in comparison with non-mutant chondrosarcomas.

\section{Expression of genes regulating metabolism correlates with prognosis in chondrosarcomas}

To determine the significance of our findings in a second chondrosarcoma patient cohort, we explored the expression levels of regulatory genes of metabolic pathways from previously published gene expression and patient survival data $[24,25]$. Using normalized microarray gene expression data $[24,25]$, we found a number of genes involved in various metabolic processes to be elevated in mutant IDH tumors (Supplemental Figure $3 \mathrm{~A})$. For instance, genes involved in the regulation of glutamine metabolism, GLS and GLUD1; a biomarker of glycolysis, $L D H A$; and genes involved in TCA cycle regulation, $P D K 1, P D H B, M D H 1$, and $S H M T 2$; were all significantly elevated in mutant $I D H$ chondrosarcomas compared with non-mutant tumors (Supplemental Figure $3 \mathrm{~A})$.
The expression levels of a number of these genes significantly correlated with patient survival when analyzed using Kaplan-Meier survival analysis (Supplemental Figure 3B-H). Interestingly, chondrosarcomas with high expression levels of two regulators of acylcarnitine metabolism, CPT1A and FASN, displayed worse patient outcome (Supplemental Figure 3B-C). High expression of regulatory genes of the TCA cycle (SDHAF2, MDH1, $M D H 2$, and $P D H B)$ and glutamine metabolism (GLUD1), were also predictive of patient outcome (Supplemental Figure 3E-H, D). Taken together, these data are constant with the notion that specific metabolites and their associated regulatory genes are critical in chondrosarcoma metabolomics, drive tumor aggressiveness, and are predictive of patient survival.

\section{Discussion}

Here we report metabolic differences between mutant IDH chondrosarcomas and non-mutant chondrosarcomas across a spectrum of organic acids, amino acids, and acylcarnitines measured by targeted GC/MS and MS/MS mass spectrometry. Mutant IDH chondrosarcomas display metabolomes enriched for lactate, late-stage TCA cycle intermediates, multiple amino acids, and a subset of acylcarnitine species. Interestingly, UMAP projection analysis of our metabolite dataset revealed that organic acids and amino acids displayed spatially strong and distinct clustering patterns in accordance with each chondrosarcoma genotype. Upon analysis of all 69 metabolites by UMAP, this revealed spatially distinct clustering in mutant $I D H$ chondrosarcomas and non-mutant chondrosarcomas. Thus, from our complete dataset, we were also able to conclude that mutant $I D H$ chondrosarcomas display a distinct metabolic profile compared with non-mutant chondrosarcomas.

Metabolic changes can be cell type dependent, and although other studies show metabolic changes due to IDH mutations in other cell types, data from these 
studies do not extend to all cell types. Metabolic changes in cell culture may not reflect changes seen in vivo. This is a particular issue in certain tumor types like chondrosarcomas, where the cell phenotype changes in vitro. Indeed, differences in diet, time of surgery, and the presence of medical comorbidities will cause metabolic changes in tumors. To control for these factors while still analyzing tumors in vivo, we studied xenografts, allowing us to control for various external factors. Thus, our approach allows an analysis of chondrosarcoma tumor metabolism eliminating confounding factors of cell culture and patient variability.

We found an elevation of lactate in mutant IDH chondrosarcomas. Conversion of glucose carbon to lactate by the lactate dehydrogenase ( $L D H A)$ enzyme is a classic signature of cancer metabolism [43]. More importantly, lactate is not only a metabolic by-product of active glycolysis but has many other consequential effects on cancer bioenergetics and carcinogenesis [43]. Glycolytic tumors that produce lactate create an acidic tumor microenvironment which supports metastasis, angiogenesis, and immunosuppression [44]. It has also been demonstrated that neoplastic cells possess the ability to import lactate into the mitochondria to fuel energy production [45]. While a prior study showed elevated $L D H A$ activity in chondrosarcomas, our data shows that genotype alters the level of lactate, and as such genotype should be taken into account when considering strategies to target this pathway [46].

Hypoxic tumors that have the ability to thrive in low oxygen environments are associated with lactate accumulation [43, 47]. Interestingly, it has been reported that glucose metabolism and hypoxia-inducible factor (HIF1a) levels are elevated in chondrosarcomas and correlated with a higher pathological grade and lower patient survival rate [48]. This suggests that mutant $I D H$ chondrosarcomas possess a greater dependence on glycolysis for energy and are metabolically more active compared with non-mutant chondrosarcomas.

Another metabolic alteration caused by a mutated $I D H$ enzyme was the broad elevation of amino acids. Specifically, amino acid profiling from our study revealed that glutamine and glutamate were abundant amino acids in chondrosarcomas and were elevated in mutant IDH chondrosarcomas, suggesting that there is increased dependence on glutaminolysis. This metabolic alteration has been identified in mutant $I D H$ solid tumors [4, 37-39] and has led to two clinical trials targeting inhibition of glutaminolysis. Thus, our amino acid profile of mutant IDH chondrosarcomas confirms elevated levels of glutamine and glutamate and the notion that a greater dependence on glutaminolysis is required to support energy production in chondrosarcoma.
Similar to our findings in chondrosarcomas, mutant $I D H$ gliomas also show a global elevation of free amino acids and lipid precursors [49]. While findings in gliomas show depletion of the late-stage TCA cycle intermediates, fumarate and malate [49], we did not observe this in chondrosarcoma. This finding could be a result of enhanced amino acid metabolism aiding in the replenishment of late-stage TCA cycle intermediates and maintenance of carbon cycling in mutant $I D H$ chondrosarcomas. Fumarate is replenished by phenylalanine and tyrosine, both of which are amino acids elevated in $I D H$ mutant chondrosarcomas, and malate is derived from pyruvate, an organic acid which also showed a trend for increase in mutant IDH chondrosarcomas. Moreover, opposing metabolic findings from Reitman et al. may be explained due to a different cell type being profiled and the in vitro conditions of their study [49].

We found distinct acylcarnitine derangements in mutant IDH versus non-mutant chondrosarcomas. Our analysis suggested that lipid and amino acid-derived acylcarnitine species were elevated in mutant $I D H$ chondrosarcomas. This could suggest that in response to mutation of the $I D H$ enzyme, chondrosarcomas resort to a greater dependence on fatty acid oxidation and amino acid metabolism to maintain tumor cell energetics. Studies in skeletal muscle reported that accumulation of mitochondrial acylcarnitine species can also be a signature of mitochondrial lipid overload or ineffective mitochondrial lipid catabolism [14]. Thus, it remains unclear if the abundant acylcarnitine levels in mutant IDH chondrosarcomas are a signature of enhanced influx of acylcarnitines in the mitochondria from increased mitochondrial fatty acid catabolism or from "incomplete" fatty acid oxidation. To investigate this phenomenon more comprehensively, a future point of interest would be to assess mutant $I D H$ chondrosarcoma mitochondria $\beta$-oxidative capacity and acylcarnitine flux metabolism by carbon isotope labeling.

Future directions of study include addressing the elevation of specific amino acid species and their associated roles in neoplastic biochemical pathways. For instance, glycine was the most abundant amino acid in chondrosarcomas and was elevated in mutant IDH chondrosarcomas. Serine was also found in elevated levels in mutant IDH chondrosarcomas. Glycine and serine are biosynthetically linked and aid in the synthesis of proteins, nucleic acids, and lipids crucial to cancer cell growth $[50,51]$. Investigation of the association of serine and glycine with tumorigenesis and malignancy [50,51] is an attractive candidate for future studies in mutant IDH chondrosarcomas.

Our study also revealed elevated BCAAs (valine, leucine, and isoleucine) levels in mutant $I D H$ chondrosarcomas. BCAAs were recently found to be a source of 
carbon for fatty acid biosynthesis in neoplastic proliferation, and suppression of BCAA metabolism led to a reduction in fatty acid levels [42, 52]. This interesting interplay between BCAA metabolism and fatty acid metabolism is another attractive candidate for further investigation in mutant $I D H$ chondrosarcomas.

\section{Conclusions}

Our metabolomics profiling of 17 human chondrosarcomas reveals a number of distinct alterations in mutant $I D H$ chondrosarcomas including differences in glycolytic intermediates, TCA cycle intermediates, amino acids, and acylcarnitines levels. This study revealed that mutant $I D H$ chondrosarcomas are metabolically more active tumors compared with $I D H$ non-mutant chondrosarcomas. While there are conflicting data on $I D H$ mutational status and associated clinical outcome in chondrosarcomas [53, 54], our metabolomic profiling shows that mutant IDH chondrosarcomas are metabolically distinct and more active than non-mutant chondrosarcomas, giving rise to the potential that $I D H$ mutational state alters cell behavior. The global elevation of amino acids, lactate accumulation, and elevation of acylcarnitines and eventual metabolic dependence of these metabolites for neoplastic energetics is a signature of IDH mutant chondrosarcomas. In support of our metabolomics findings, our analysis of previously published mRNA microarray data $[24,25]$ revealed increased gene expression levels of specific metabolism genes in mutant $I D H$ chondrosarcomas, which was also found to correlate to poor patient survival.

\begin{abstract}
Abbreviations
IDH1: Isocitrate dehydrogenase 1; IDH2: Isocitrate dehydrogenase 2; UMAP: Uniform Manifold Approximation and Projection; TCA: Tricarboxylic acid; a-KG: a-Ketoglutarate; D-2HG: D-2-Hydroxyglutarate; HIF1a: Hypoxiainducible factor 1a; GC/MS: Gas chromatography-mass spectrometry; MS/ MS: Mass spectrometry; IRB: Institutional review board; BCAAs: Branchedchain amino acids; LDHA: Lactate dehydrogenase
\end{abstract}

\section{Supplementary Information}

The online version contains supplementary material available at https://doi. org/10.1186/s40170-021-00247-8.

Additional file 1: Supplemental Figure 1. UMAP clustering analysis of metabolites from mutant IDH and non-mutant chondrosarcomas display strong spatial clustering in organic acid and amino acid groups but weak spatial clustering in acylcarnitine species. UMAP analysis was performed in each metabolite group A) 7 organic acids (5 TCA cycle and 2 glycolytic intermediates, B) 17 amino acids, C) 45 acylcarnitines, D) total 69 metabolites

Additional file 2: Supplemental Figure 2. ${ }^{13} \mathrm{C}$ a-KG labelling is reduced in mutant $I D H 1$ and $I D H 2$ chondrosarcoma cells based on measurements of citrate and pyruvate labelling. ${ }^{13} \mathrm{C}$ isotope labelling of $\mathrm{a}-\mathrm{KG}$ was achieved by 6 hours $\left[{ }^{13} \mathrm{C}_{6}\right]$ labelling of glucose. Data normalized from citrate (M2) and pyruvate (M3) labelling display effects of mutated IDH1 and $I D H 2$ enzymes $A$ ) reduction of a-KG carbon labelling from M2 citrate in mutant IDH chondrosarcoma cells B) reduction of a-KG carbon labelling from M3 pyruvate in mutant IDH chondrosarcoma cells
Additional file 3: Supplemental Figure 3. Expression of genes regulating metabolism correlates with prognosis in chondrosarcomas. A number of metabolism genes were found to be A) elevated in mutant IDH1 and/or mutant IDH2 chondrosarcomas and $\mathrm{B}-\mathrm{H}$ ) were predicative of patient survival

\section{Acknowledgements}

We thank Qingxia Wei for the generation of xenografts from patient chondrosarcoma tissues. We also thank our funding sources: NIH R01 AR066765 and CIHR: MOP-37913 for funding this study.

\section{Authors' contributions}

SP, BAA, CBN, and JSW conceived the idea of the study and designed the study. SP, OI, AKHL, HHZ, GZ, and CBN acquired the data from the study. SP and $C B N$ analyzed and interpreted data from the study. JTM analyzed a portion of the data from the study. SP, BAA, CBN, and JSW drafted and revised the work. The author(s) read and approved the final manuscript.

\section{Funding}

NIH R01 AR066765, CIHR: MOP-37913

Availability of data and materials

All data generated or analyzed during this study are included in this published article (and its supplementary information files).

\section{Declarations}

Ethics approval and consent to participate

The following study (01-0138-U) has received continued approval from the Mount Sinai Hospital Research Ethics Board for the usage of human samples for research. Human samples were handled and animal work complied with the IRB guidelines.

\section{Consent for publication}

Not applicable

\section{Competing interests}

The authors declare that they have no competing interests.

\section{Author details}

'Developmental and Stem Cell Biology, Hospital for Sick Children, Toronto, ON, Canada. ${ }^{2}$ Institute of Medical Science, University of Toronto, Toronto, ON, Canada. ${ }^{3}$ Department of Pharmacology \& Cancer Biology, Duke University, Durham, NC, USA. ${ }^{4}$ Sarah W. Stedman Nutrition and Metabolism Center and Duke Molecular Physiology Institute, Duke University Medical Center,

Durham, NC, USA. ${ }^{5}$ Department of Orthopaedic Surgery, Duke University, 311 Trent, Durham, NC 27710, USA. ' Lunenfeld-Tanenbaum Research Institute, and the University Musculoskeletal Oncology Unit, Mount Sinai Hospital, Toronto, ON, Canada.

Received: 2 September 2020 Accepted: 3 March 2021

Published online: 24 March 2021

\section{References}

1. Hanahan D, Weinberg RA. Hallmarks of cancer: the next generation. Cell. 2011;144(5):646-74. https://doi.org/10.1016/j.cell.2011.02.013.

2. Hirata M, Sasaki M, Cairns RA, Inoue S, Puviindran V, Li WY, Snow BE, Jones LD, Wei Q, Sato S, Tang YJ, Nadesan P, Rockel J, Whetstone H, Poon R, Weng A, Gross S, Straley K, Gliser C, Xu Y, Wunder J, Mak TW, Alman BA. Mutant IDH is sufficient to initiate enchondromatosis in mice. Proc Natl Acad Sci. 2015;112(9):2829-34. https://doi.org/10.1073/pnas.1424400112.

3. Amary MF, Bacsi K, Maggiani F, Damato S, Halai D, Berisha F, Pollock R, O'Donnell P, Grigoriadis A, Diss T, Eskandarpour M, Presneau N, Hogendoorn PCW, Futreal A, Tirabosco R, Flanagan AM. IDH1 and IDH2 mutations are frequent events in central chondrosarcoma and central and periosteal chondromas but not in other mesenchymal tumours. J Pathol. 2011;224(3):334-43. https://doi.org/10.1002/path.2913.

4. Peterse EFP, Niessen $B$, Addie RD, de Jong $Y$, Cleven AHG, Kruisselbrink AB, van den Akker BEWM, Molenaar RJ, Cleton-Jansen AM, Bovée JVMG. Targeting glutaminolysis in chondrosarcoma in context of the IDH1/2 
mutation. Br J Cancer. 2018;118(8):1074-83. https://doi.org/10.1038/s41416018-0050-9.

5. Turkalp Z, Karamchandani J, Das S. IDH mutation in glioma: New insights and promises for the future. JAMA Neurol. 2014;71(10):1319-25. https://doi. org/10.1001/jamaneurol.2014.1205.

6. Borger DR, Goyal L, Yau T, et al. Circulating Oncometabolite 2Hydroxyglutarate Is a Potential Surrogate Biomarker in Patients with Isocitrate Dehydrogenase-Mutant Intrahepatic Cholangiocarcinoma. Clin Cancer Res. 2014;20(7):1884-90. https://doi.org/10.1158/1078-0432.CCR-13-2 649.

7. Parker SJ, Metallo CM. Metabolic consequences of oncogenic IDH mutations. Pharmacol Ther. 2015;152:54-62. https://doi.org/10.1016/j.pha rmthera.2015.05.003.

8. Kickingereder P, Sahm F, Radbruch A, Wick W, Heiland S, Deimling A, Bendszus $\mathrm{M}$, Wiestler $\mathrm{B}$. IDH mutation status is associated with a distinct hypoxia/angiogenesis transcriptome signature which is non-invasively predictable with rCBV imaging in human glioma. Sci Rep. 2015;5(July):1-9. https://doi.org/10.1038/srep16238.

9. Metallo CM, Gameiro PA, Bell EL, Mattaini KR, Yang J, Hiller K, Jewell CM, Johnson ZR, Irvine DJ, Guarente L, Kelleher JK, Vander Heiden MG, lliopoulos $\mathrm{O}$, Stephanopoulos G. Reductive glutamine metabolism by IDH1 mediates lipogenesis under hypoxia. Nature. 2012;481(7381):380-4. https://doi.org/1 0.1038 /nature 10602 .

10. Newgard CB. Metabolomics and Metabolic Diseases: Where Do We Stand? Cell Metab. 2017;25(1):43-56. https://doi.org/10.1016/j.cmet.2016.09.018.

11. Dai C, Arceo J, Arnold J, Sreekumar A, Dovichi NJ, Li J, Littlepage LE. Metabolomics of oncogene-specific metabolic reprogramming during breast cancer. Cancer Metab. 2018;6(1):1-17. https://doi.org/10.1186/s40170018-0175-6.

12. Fujisawa K, Takami T, Okada S, Hara K, Matsumoto T, Yamamoto N, Yamasaki T, Sakaida I. Analysis of Metabolomic Changes in Mesenchymal Stem Cells on Treatment with Desferrioxamine as a Hypoxia Mimetic Compared with Hypoxic Conditions. Stem Cells. 2018;36(8):1226-36. https://doi.org/10.1002/ stem.2826.

13. Moreno $P$, Jiménez-Jiménez $C$, Garrido-Rodríguez M, Calderón-Santiago M, Molina S, Lara-Chica M, Priego-Capote F, Salvatierra Á, Muñoz E, Calzado MA. Metabolomic profiling of human lung tumor tissues - nucleotide metabolism as a candidate for therapeutic interventions and biomarkers. Mol Oncol. 2018;12(10):1778-96. https://doi.org/10.1002/1878-0261.12369.

14. Koves TR, Ussher JR, Noland RC, Slentz D, Mosedale M, Ilkayeva O, Bain J, Stevens R, Dyck JRB, Newgard CB, Lopaschuk GD, Muoio DM. Mitochondrial Overload and Incomplete Fatty Acid Oxidation Contribute to Skeletal Muscle Insulin Resistance. Cell Metab. 2008;7(1):45-56. https://doi.org/10.101 6/j.cmet.2007.10.013

15. Bain JR, Stevens RD, Wenner BR, Ilkayeva O, Muoio DM, Newgard CB. Metabolomics applied to diabetes research: Moving from information to knowledge. Diabetes. 2009;58(11):2429-43. https://doi.org/10.2337/db09-0580.

16. An J, Muoio DM, Shiota M, Fujimoto Y, Cline GW, Shulman GI, Koves TR, Stevens R, Millington D, Newgard CB. Hepatic expression of malonyl-CoA decarboxylase reverses muscle, liver and whole-animal insulin resistance. Nat Med. 2004:10(3):268-74. https://doi.org/10.1038/nm995.

17. Ferrara $C T$, Wang $P$, Neto $E C$, et al. Genetic networks of liver metabolism revealed by integration of metabolic and transcriptional profiling. PLoS Genet. 2008;4(3). https://doi.org/10.1371/journal.pgen.1000034.

18. Jensen MV, Joseph JW, Ilkayeva O, Burgess S, Lu D, Ronnebaum SM, Odegaard M, Becker TC, Sherry AD, Newgard CB. Compensatory responses to pyruvate carboxylase suppression in islet $\beta$-cells: Preservation of glucosestimulated insulin secretion. J Biol Chem. 2006;281(31):22342-51. https://doi. org/10.1074/jbc.M604350200

19. Mclnnes L, Healy J, Melville J. UMAP: Uniform Manifold Approximation and Projection for Dimension Reduction. arXiv e-prints. 2018.

20. Team R. R: A Language and Environment for Statistical Computing. R Foundation for Statistical Computing; 2020.

21. Wang Y, Christopher BA, Wilson KA, Muoio D, McGarrah RW, Brunengraber $H$, Zhang GF. Propionate-induced changes in cardiac metabolism, notably CoA trapping, are not altered by l-carnitine. Am J Physiol Endocrinol Metab. 2018;315(622-633):E622-33. https://doi.org/10.1152/ajpendo.00081.2018.

22. Fernandez CA, Des Rosiers C, Previs SF, David F, Brunengraber H. Correction of $13 \mathrm{C}$ mass isotopomer distributions for natural stable isotope abundance. J Mass Spectrom. 1996;31(3):255-62. https://doi.org/10.1002/(SICl)10969888(199603)31:3<255::AID-JMS290>3.0.CO;2-3.
23. Tomcik K, Ibarra RA, Sadhukhan S, Han Y, G.P. Tochtrop GFZ. Isotopomer enrichment assay for very short chain fatty acids and its metabolic applications. Anal Biochem. 2011;410(1):110-7. https://doi.org/10.1016/j.ab.2 010.11.030.

24. Nicolle R, Ayadi M, Gomez-Brouchet A, Armenoult L, Banneau G, Elarouci N, Tallegas M, Decouvelaere AV, Aubert S, Rédini F, Marie B, Labit-Bouvier C, Reina N, Karanian M, le Nail LR, Anract P, Gouin F, Larousserie F, de Reyniès A, de Pinieux G. Integrated molecular characterization of chondrosarcoma reveals critical determinants of disease progression. Nat Commun. 2019; 10(1):1-11. https://doi.org/10.1038/s41467-019-12525-7.

25. Nabila E. E-MTAB-7265 - High-throughput sequencing of miRNA of cartilage tumors. 2018. https://www.ebi.ac.uk/arrayexpress/experiments/E-MTAB-72 $65 /$.

26. European Bioinformatics Institute database (EMBL-EBI).

27. Badur M, Muthusamy T, Parker S, et al. Oncogenic R132 IDH1 Mutations Limit NADPH for De Novo Lipogenesis through (D)2-Hydroxyglutarate Production in Fibrosarcoma Cells. Cell Rep. 2018;25(4).

28. Zhang $Y$, Lv W, Li Q, et al. IDH2 compensates for IDH1 mutation to maintain cell survival under hypoxic conditions in IDH1-mutant tumor cells. Spandidos Publ. 2019;20(2)

29. Jiang L, Shestov AA, Swain P, et al. Reductive carboxylation supports redox homeostasis during anchorage-independent growth. Nature. 2016; 7598(532). https://doi.org/10.1530/ERC-14-0411.Persistent.

30. Fendt SM, Bell EL, Keibler MA, Olenchock BA, Mayers JR, Wasylenko TM, Vokes NI, Guarente L, Heiden MGV, Stephanopoulos G. Reductive glutamine metabolism is a function of the a-ketoglutarate to citrate ratio in cells. Nat Commun. 2013;4(1):1-11. https://doi.org/10.1038/ncomms3236.

31. Mullen AR, Wheaton WW, Jin ES, et al. Reductive carboxylation supports growth in tumor cells with defective mitochondria. Nature. 2012;481(7381): 385-8. https://doi.org/10.1038/nature10642.Reductive.

32. Raimundo N, Baysal BE, Shadel GS. Revisiting the TCA cycle: Signaling to tumor formation. Trends Mol Med. 2011;17(11):641-9. https://doi.org/10.101 6/j.molmed.2011.06.001.

33. Yang C, Ko B, Hensley CT, Jiang L, Wasti AT, Kim J, Sudderth J, Calvaruso MA, Lumata L, Mitsche M, Rutter J, Merritt ME, DeBerardinis RJ. Glutamine oxidation maintains the TCA cycle and cell survival during impaired mitochondrial pyruvate transport. Mol Cell. 2014;56(3):414-24. https://doi. org/10.1016/j.molcel.2014.09.025

34. Kimmelman AC, White E. Autophagy and Tumor Metabolism. Cell Metab. 2017;25(5):1037-43. https://doi.org/10.1016/j.cmet.2017.04.004.

35. Mathew R, Karantza-Wadsworth V, White E. Role of autophagy in cancer. Nat Rev Cancer. 2007;7(December):961-7. https://doi.org/10.1038/nrc2254. Role.

36. Russell RC, Yuan HX, Guan KL. Autophagy regulation by nutrient signaling. Cell Res. 2014;24(1):42-57. https://doi.org/10.1038/cr.2013.166

37. Bonfili L, Cecarini V, Cuccioloni M, et al. Essential amino acid mixtures drive cancer cells to apoptosis through proteosome inhibition and autophagy activation. 2017;38(1):42-9. https://doi.org/10.1111/ijlh.12426.

38. Vettore L, Westbrook RL, Tennant DA. New aspects of amino acid metabolism in cancer. Br J Cancer. 2019;(June). https://doi.org/10.1038/ s41416-019-0620-5.

39. Hiraoka N, Toue S, Okamoto C, Kikuchi S, Ino Y, Yamazaki-Itoh R, Esaki M, Nara S, Kishi Y, Imaizumi A, Ono N, Shimada K. Tissue amino acid profiles are characteristic of tumor type, malignant phenotype, and tumor progression in pancreatic tumors. Sci Rep. 2019;9(1):1-14. https://doi.org/1 0.1038/s41598-019-46404-4.

40. Sullivan LB, Luengo A, Danai LV, Bush LN, Diehl FF, Hosios AM, Lau AN, Elmiligy S, Malstrom S, Lewis CA, Vander Heiden MG. Aspartate is an endogenous metabolic limitation for tumour growth. Nat Cell Biol. 2018; 20(7):782-8. https://doi.org/10.1038/s41556-018-0125-0.

41. Sullivan LB, Gui DY, Hosios AM, Bush LN, Freinkman E, Vander Heiden MG Supporting Aspartate Biosynthesis Is an Essential Function of Respiration in Proliferating Cells. Cell. 2015;162(3):552-63. https://doi.org/10.1016/j.cell.201 5.07.017.

42. Ananieva EA, Wilkinson AC. Branched-chain amino acid metabolism in cancer. Curr Opin Clin Nutr Metab Care. 2018;21(1):64-70. https://doi.org/1 0.1097/MCO.0000000000000430.

43. San-Millán I, Brooks GA. Reexamining cancer metabolism: Lactate production for carcinogenesis could be the purpose and explanation of the Warburg Effect. Carcinogenesis. 2017;38(2):119-33. https://doi.org/10.1093/ carcin/bgw127. 
44. de la Cruz-López KG, Castro-Muñoz LJ, Reyes-Hernández DO, GarcíaCarrancá A, Manzo-Merino J. Lactate in the Regulation of Tumor Microenvironment and Therapeutic Approaches. Front Oncol. 2019; 9(November). https://doi.org/10.3389/fonc.2019.01143.

45. Kane DA. Lactate oxidation at the mitochondria: A lactate-malate-aspartate shuttle at work. Front Neurosci. 2014;8(OCT):1-6. https://doi.org/10.3389/ fnins.2014.00366

46. Hua G, Liu Y, Li X, XU P, Luo Y. Targeting glucose metabolism in chondrosarcoma cells enhances the sensitivity to doxorubicin through the inhibition of lactate dehydrogenase-A. Oncol Rep. 2014;31(6):2727-34. https://doi.org/10.3892/or.2014.3156.

47. Jiang B. Aerobic glycolysis and high level of lactate in cancer metabolism and microenvironment. Genes Dis. 2017;4(1):25-7. https://doi.org/10.1016/j. gendis.2017.02.003

48. Chen C, Zhou H, Wei F, Jiang L, Liu X, Liu Z, Ma Q. Increased levels of hypoxia-inducible factor-1a are associated with $\mathrm{BCl}-\mathrm{xL}$ expression, tumor apoptosis, and clinical outcome in chondrosarcoma. J Orthop Res. 2011; 29(1):143-51. https://doi.org/10.1002/jor.21193.

49. Reitman ZJ, Jin G, Karoly ED, Spasojevic I, Yang J, Kinzler KW. Profiling the effects of isocitrate dehydrogenase 1 and 2 mutations on the cellular metabolome; 2011. https://doi.org/10.1073/pnas.1019393108/-/ DCSupplemental.www.pnas.org/cgi/doi/10.1073/pnas.1019393108

50. Amelio I, Cutruzzolá F, Antonov A, Agostini M, Melino G. Serine and glycine metabolism in cancer. Trends Biochem Sci. 2014;39(4):191-8. https://doi. org/10.1016/j.tibs.2014.02.004.

51. Braun JE, Huntzinger $E$, Fauser $M$, et al. Metabolite Profiling Identifies a Key Role for Glycine in Rapid Cancer Cell Proliferation. Science. 2012; (May):1040-5

52. Lee JH, Cho Y r, Kim JH, et al. Branched-chain amino acids sustain pancreatic cancer growth by regulating lipid metabolism. Exp Mol Med. 2019;51(11). https://doi.org/10.1038/s12276-019-0350-z.

53. Cleven AHG, Suijker J, Agrogiannis $G$, Briaire-de Bruijn IH, Frizzell N, Hoekstra AS, Wijers-Koster PM, Cleton-Jansen AM, Bovée JVMG. IDH1 or -2 mutations do not predict outcome and do not cause loss of 5-hydroxymethylcytosine or altered histone modifications in central chondrosarcomas. Clin Sarcoma Res. 2017;7(1):1-10. https://doi.org/10.1186/s13569-017-0074-6.

54. Zhu GG, Nafa K, Agaram N, Zehir A, Benayed R, Sadowska J, Borsu L, Kelly C, Tap WD, Fabbri N, Athanasian E, Boland PJ, Healey JH, Berger MF, Ladanyi M, Hameed M. Genomic profiling identifies association of IDH1/IDH2 mutation with longer relapse-free and metastasis-free survival in high-grade chondrosarcoma. Clin Cancer Res. 2020;26(2):419-27. https://doi.org/10.11 58/1078-0432.CCR-18-4212

\section{Publisher's Note}

Springer Nature remains neutral with regard to jurisdictional claims in published maps and institutional affiliations.

Ready to submit your research? Choose BMC and benefit from:

- fast, convenient online submission

- thorough peer review by experienced researchers in your field

- rapid publication on acceptance

- support for research data, including large and complex data types

- gold Open Access which fosters wider collaboration and increased citations

- maximum visibility for your research: over $100 \mathrm{M}$ website views per year

At BMC, research is always in progress.

Learn more biomedcentral.com/submissions 